\title{
The Application Value of Serum HE4 in the Diagnosis of Lung Cancer
}

Yuhui Wang, Zhenming Wang, Yansheng Ding*, Fengqiang Sun, Xiaomei Ding

\begin{abstract}
Background: To investigate the clinical value of $H E 4$ detection in the diagnosis of lung cancer and the clinical significance of combined detection with CEA, NSE and CYFRA21-1. Methods: 90 cases of lung cancer, 30 cases of pulmonary tuberculosis, 30 cases of pneumonia and 30 cases of health physical examination were selected. The levels of serum HE4, CYFRA21-1, CEA and NSE were detected by electrochemiluminescence method. Statistical analysis was performed to observe the sensitivity and specificity. Results: The levels of serum HE4, CEA, NSE and CYFRA21-1 in lung cancer group were significantly higher than those in tuberculosis group and health physical examination group. There was no significant difference in the levels of HE4, CEA and NSE between the lung cancer group and the pneumonia group, the difference of CYFRA21-1 level was statistically significant $(\mathrm{p}<0.05)$. With health physical examination group as normal controls, the sensitivity and specificity of combined detection of HE4, CEA, NSE and CYFRA21-1 in the diagnosis of lung cancer were $82.2 \%$ and $90.0 \%$, and the area under the curve (AUC) was 0.907 , followed by $H E 4$ (0.867), CYFRA21-1 (0.787), CEA (0.752) and NSE (0.747). Conclusion: HE4 can be used as a serological marker for the diagnosis of lung cancer. The combined detection of HE4, CEA, NSE and CYFRA21-1 can improve the diagnosis of lung cancer. Serum $H E 4$ levels are highly specific in distinguishing between lung cancer patients and normal population, and are equivalent to CYFRA21-1; but they are less specific than CYFRA21-1 in distinguishing lung cancer patients from pneumonia patients.
\end{abstract}

Keywords: HE4- CYFRA21-1-CEA- NSE- lung cancer

Asian Pac J Cancer Prev, 20 (8), 2405-2407

\section{Introduction}

Clinically, the diagnostic value of serum tumor markers has been confirmed, and markers such as carcinoembryonic antigen $(C E A)$, neuron-specific enolase (NSE), and cytokeratin 19 fragment (CYFRA21-1) have been widely used for the diagnosis of lung cancer. Human epididymis secretory protein 4 (HE4) is a new tumor marker, highly expressed in ovarian cancer, and extremely low in normal tissues. It is clinically used for early diagnosis of ovarian cancer. In recent years, it has been reported in the literature that HE4 can also be used for early diagnosis of lung cancer (Mo et al., 2018; Nagy, 2014). This study was to investigate the value of $H E 4$ detection in the diagnosis of lung cancer and the clinical significance of combined detection with CEA, NSE and CYFRA21-1.

\section{Materials and Methods}

\section{Sample collection}

From January 2016 to October 2018, there were 90 patients with lung cancer in Weifang People's Hospital, including 56 males and 34 females, aged 46-81
(61.0 \pm 12.8$)$ years old, including 29 cases of squamous cell carcinoma, 50 cases of adenocarcinoma, 9 cases of small cell lung cancer and 2 cases of large cell lung cancer. All cases were confirmed by pathology, and no surgery, radiotherapy and chemotherapy were performed before blood samples were collected. Another 30 patients with pneumonia in Weifang People's Hospital were selected, including 14 males and 16 females, aged 44-89 (67.0 \pm 17.5$)$ years old; 30 health physical examination persons as normal controls, including 15 males and 15 females, aged 45-77 ( 58.8 \pm 10.9 ) years old. 30 patients with pulmonary tuberculosis in Weifang City Respiratory Hospital were selected, including 17 males and 13 females, aged 30-68 (39.0 \pm 8.5$)$ years old.

\section{Detection method}

All selected subjects were collected $5 \mathrm{ml}$ of peripheral venous blood on an empty stomach, left at room temperature for about $1 \mathrm{~h}$, centrifuged at $4,000 \times \mathrm{g}$ for $5 \mathrm{~min}$, separated serum, and stored in a refrigerator at $-80^{\circ} \mathrm{C}$ for use. HE4, CEA, NSE and CYFRA21-1 were detected by Roche Cobas e601 electrochemiluminescence immunoassay analyzer. The reagents were supplied by Roche and the reagents were calibrated before the 
experiment.

\section{Statistical analysis}

The results of the measurement data were expressed as median (quartile). The Mann-Whitney U rank sum test was used for comparison between groups, the difference was statistically significant at $\mathrm{P}<0.05$. Data comparison and receiver operating characteristic curve (ROC) were statistically analyzed using SPSS 21.0 software. The sensitivity, specificity, and area under the curve (AUC) were calculated from the respective ROC curves.

\section{Results}

Serum levels of HE4, CEA, NSE, and CYFRA 21-1 in each group. The results are shown in Table 1.

The levels of serum HE4, CEA, NSE and CYFRA21-1 in lung cancer group were significantly higher than those in tuberculosis group and health physical examination group $(\mathrm{P}<0.05)$. There were no significant differences in $H E 4, C E A$, and NSE levels between the lung cancer group and the pneumonia group $(\mathrm{p}=0.844, \mathrm{p}=0.087, \mathrm{p}=0.687$ ), and the CYFRA21-1 level was statistically significant $(\mathrm{p}<0.05)$.

Diagnostic efficiency of HE4, CEA, NSE and CYFRA21-1 for lung cancer

Taking health physical examination group as normal controls, with $76.885 \mathrm{pmol} / \mathrm{L}$ as the optimal cut-off value, the sensitivity of HE4 for diagnosis of lung cancer was $66.7 \%$, and the specificity was $96.7 \%$. With $3.25 \mathrm{ug} / \mathrm{L}$ as the optimal cut-off value, the sensitivity of $C E A$ for diagnosis of lung cancer was $61.1 \%$, and the specificity was $80.0 \%$. With $11.01 \mathrm{ng} / \mathrm{ml}$ as the optimal cut-off value, the sensitivity of NSE for diagnosis of lung cancer was $64.4 \%$, and the specificity was $86.7 \%$. With $3.23 \mathrm{ng} / \mathrm{ml}$ as the optimal cut-off value, the sensitivity of CYFRA21-1 for diagnosis of lung cancer was $58.9 \%$, and the specificity was $86.7 \%$. The sensitivity of combined detection of HE4, $C E A, N S E$, and CYFRA21-1 for diagnosis of lung cancer was $82.2 \%$ and the specificity was $90.0 \%$. ROC AUC showed that the combined detection of HE4, CEA, NSE and CYFRA21-1 had the largest $A U C$ (0.907), followed by HE4 (0.867), CYFRA21-1 (0.787), CEA ( 0.752) and NSE (0.747) See Figure 1.

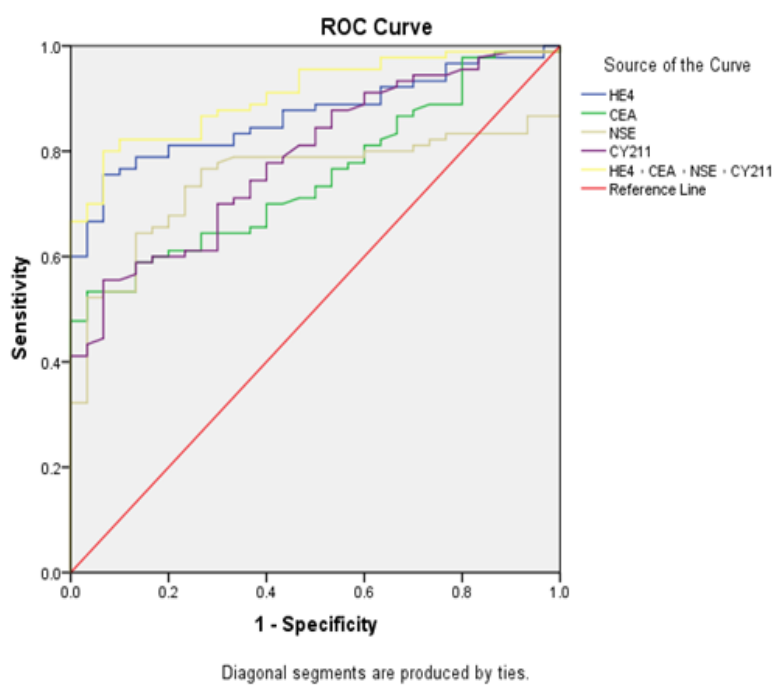

Figure 1. The Diagnostic Efficiency of HE4 and Other Markers for Lung Cancer, with Health Physical Examination Group as Normal Controls

\section{Discussion}

$C E A$ is a complex soluble glycoprotein originally discovered in colon cancer and fetal intestinal tissue. Tumors of various organs can cause elevated levels of serum $C E A$. Serum $C E A$ levels have important diagnostic value for lung cancer, especially lung adenocarcinoma. $N S E$ is a protease secreted by neurogenic cells, which is markedly elevated in neuroendocrine tumors and small cell lung cancer. NSE can be used as a marker for the detection of small cell lung cancer. NSE can also be elevated in patients with non-small cell lung cancer, but its sensitivity is low. CYFRA 21-1 is a soluble fragment of cytokeratin 19 expressed in epithelial-derived malignant cells, and the serum CYFRA21-1 levels will increase when cells are lysed. CYFRA 21-1 is the marker for non-small cell lung cancer, and the positive rate of lung squamous cell carcinoma is higher than that of lung adenocarcinoma. A number of studies have shown that CYFRA 21-1 has a relatively high sensitivity and specificity for the diagnosis of lung cancer compared with other classical tumor markers, which is consistent with the statistical results of this study. Single tumor markers do not have sufficient diagnostic efficiency for the diagnosis of lung cancer, especially the sensitivity is not high enough.

HE4 was originally discovered by Kiechhoff et al.,

Table 1. Serum Levels of HE4, CEA, NSE and CYFRA21-1 in Each Group (Median, Quartile)

\begin{tabular}{lccccc}
\hline Group & $\mathrm{n}$ & HE4 $(\mathrm{pmol} / \mathrm{L})$ & $C E A(\mathrm{ug} / \mathrm{L})$ & NSE $(\mathrm{ng} / \mathrm{ml})$ & CYFRA21-1 $(\mathrm{ng} / \mathrm{ml})$ \\
\hline Lung cancer & 90 & 88.130 & 4.135 & 13.885 & 3.845 \\
& & $(32.48 \sim 1500.00)$ & $(0.77 \sim 999.00)$ & $(1.72 \sim 289.20)$ & $(1.01 \sim 158.10)$ \\
Tuberculosis & 30 & 45.335 & 2.000 & 8.985 & 2.075 \\
& & $(31.04 \sim 283.10)$ & $(0.50 \sim 7.27)$ & $(6.57 \sim 15.44)$ & $(1.10 \sim 5.09)$ \\
Pneumonia & 30 & 78.345 & 2.765 & 11.465 & 2.410 \\
& & $(35.96 \sim 980.50)$ & $(0.89 \sim 140.70)$ & $(7.72 \sim 110.40)$ & $(1.01 \sim 123.30)$ \\
Health physical examination & 30 & 45.600 & 2.605 & 7.635 & 2.080 \\
& & $(29.70 \sim 81.51)$ & $(0.89 \sim 4.32)$ & $(5.53 \sim 18.19)$ & $(1.02 \sim 4.87)$ \\
\hline
\end{tabular}


(1991), in the epithelium of the distal epididymis, and is a member of the inhibitory protease family, encoded by the $W F D C 2$ gene. The HE4 gene is located on chromosome 20q12-13. 1 and consists of 5 exons and 4 introns. HE4 is highly expressed in the epithelium of the female reproductive system and the epididymis and vas deferens of the male reproductive system, and is a sensitive marker of ovarian cancer (Chen et al. 2014; Karlsen et al., 2015; Gislefoss et al., 2015; Ferraro et al., 2018; Lycke et al., 2018), also important for endometrial cancer (Minář et al., 2015; Hu et al., 2016; Angioli et al., 2016). As a new marker, HE4 is also highly expressed in lung cancer. Serum HE4 may be a potential marker for the diagnosis and prognosis of lung cancer and has important diagnostic value for lung cancer (Liu et al., 2013; Wang et al., 2014; Lamy et al., 2015). This study shows that the diagnostic efficiency of $H E 4$ for lung cancer is comparable to that of CYFRA 21-1, and superior to CEA and NSE.

The study found that the median of serum HE4 level was significantly higher in the pneumonia group. There was no significant difference in the levels of $H E 4, C E A$ and NSE between the lung cancer group and the pneumonia group. The difference in the level of CYFRA21-1 was statistically significant between the lung cancer group and the pneumonia group. With health physical examination group as normal controls and 76.885 $\mathrm{pmol} / \mathrm{L}$ as the optimal cut-off value, the sensitivity of HE4 in the diagnosis of lung cancer was $66.7 \%$, and the specificity was $96.7 \%$. This indicates that serum HE4 levels are highly specific in distinguishing lung cancer patients from normal people, and the specificity is not high in distinguishing lung cancer patients from pneumonia patients.

$A U C$ is used to evaluate the efficiency of the diagnostic experiment. The greater the value, the greater the diagnostic value of the experiment. From the current data, in this study, taking health physical examination group as normal controls, combined detection of HE4, CEA, NSE and CYFRA21-1 had the largest $A U C$, followed by HE4, CYFRA21-1, CEA and NSE. This indicates that combined detection of HE4,CEA, NSE and CYFRA21-1 for lung cancer is superior to single marker, especially in distinguishing between normal population and lung cancer patients, the combined detection for lung cancer has good sensitivity and specificity, which are $82.2 \%$ and $90.0 \%$ respectively.

In summary, this study shows that HE4 is a new marker of lung cancer. The diagnostic efficiency of HE4 for lung cancer is comparable to that of CYFRA 21-1, and better than $C E A$ and NSE. In distinguishing between lung cancer patients and normal population, HE4 has high specificity, but the sensitivity is slightly lower. The combined detection of HE4, CEA, NSE and CYFRA21-1 has good sensitivity and specificity. In distinguishing between lung cancer patients and pneumonia patients, the specificity of HE4 is not high, and it is not as good as CYFRA21-1.

\section{Acknowledgements}

This study was supported by grants from the weifang city health and family planning commission scientific research project plan (No. 005, 2015), and the Shandong Medical and Health Technology Development Project (2017WS063).

\section{References}

Angioli R, Capriglione S, Scaletta G, et al (2016). The role of HE4 in endometrial cancer recurrence: how to choose the optimal follow-up program. Tumour Biol, 37, 4973-8.

Chen WT, Gao X, Han XD, et al (2014). HE4 as a serum biomarker for ROMA prediction and prognosis of epithelial ovarian cancer. Asian Pac J Cancer Prev, 15, 101-5.

Ferraro S, Robbiano C, Tosca N, et al (2018). Serum human epididymis protein 4 vs. carbohydrate antigen 125 in ovarian cancer follow-up. Clin Biochem, 60, 84-90.

Gislefoss RE, Langseth H, Bolstad N, et al (2015). HE4 as an early detection biomarker of epithelial ovarian cancer: Investigations in prediagnostic specimens from the Janus serum bank. Int J Gynecol Cancer, 25, 1608-15.

$\mathrm{Hu}$ L, Du S, Guo W, et al (2016). Comparison of serum human epididymis protein 4 and carbohydrate antigen 125 as markers in endometrial cancer: A meta-analysis. Int $J$ Gynecol Cancer, 26, 331-40.

Karlsen MA, Høgdall EV, Christensen IJ, et al (2015). A novel diagnostic index combining HE4,CA125 and age may improve triage of women with suspected ovarian cancer - An international multicenter study in women with anovarian mass. Gynecol Oncol, 138, 640-6.

Kirchhoff C, Habben I, Ivell R, et al (1991). A major human epididymis specific $C D N A$ encodes a protein with sequence homology to extracellular proteinase inhibitors. Biol Reprod, 45, 350-7.

Lamy PJ, Plassot C, Pujol JL (2015). Serum HE4: An independent prognostic factor in non-small cell lung cancer. PLoS One, 10, e0128836.

Liu W, Yang J, Chi PD, et al (2013). Evaluating the clinical significance of serum HE4 levels in lung cancer and pulmonary tuberculosis. Int J Tuberc Lung Dis, 17, 1346-53.

Lycke M, Kristjansdottir B, Sundfeldt K (2018). A multicenter clinical trial validating the performance of $H E 4, C A 125$, risk of ovarian malignancy algorithm and risk of malignancy index. Gynecol Oncol, 151, 159-65.

Minář L, Klabenešová I, Jandáková E (2015).The importance of HE4 in differential diagnosis of endometrial cancer. Ceska Gynekol, 80, 256-63.

Mo D, He F (2018). Serum human epididymis secretory protein $4(H E 4)$ is a potential prognostic biomarker in non-small cell lung cancer. Clin Lab, 64, 1421-8.

Nagy B Jr, Bhattoa HP, Steiber Z, et al (2014). Human epididymis protein 4 (HE4) as a tumor marker in men with lung cancer. Clin Chem Lab Med, 52, 1639-48.

Wang X, Fan Y, Wang J, et al (2014). Evaluating the expression and diagnostic value of human epididymis protein 4 (HE4) in small cell lung cancer. Tumour Biol, 35, 6847-53.

This work is licensed under a Creative Commons AttributionNon Commercial 4.0 International License. 